\title{
Autoestima, Musicoterapia y Violencia de Género. Un encuentro grupal desde el abordaje plurimodal
}

\author{
Dra. Empar Guerrero Valverde
}

\author{
Recibido: 20/04/2019 Aceptado: 10/11/2019
}

RESUMEN. En este artículo se presentan los resultados de la intervención musicoterapéutica, llevada a cabo con 24 mujeres, víctimas de violencia de género.

Objetivos: Demostrar que la musicoterapia contribuye a: a) aumentar la autoestima de estas mujeres; b) mejorar las relaciones interpersonales; c) fomentar la iniciativa.

Método: Para las sesiones se utilizó el método de Abordaje Plurimodal de Musicoterapia. Para valorar los resultados, se aplicó antes y después, la Escala de Autoestima de Rosenberg y los IAPS de Bruscia (Los perfiles de evaluación de improvisaciones), para valorar la iniciativa y las relaciones interpersonales.

Resultados: Se observó un aumento de la autoestima en todas las mujeres, siendo más elevado en aquellas que llevaban menos tiempo, separadas de su maltratador. Asimismo, a través de los IAPS, se observó que mejoraron las relaciones interpersonales y que aumentó más la iniciativa, en las mujeres, que llevaban más tiempo separadas de su maltratador.

Palabras clave: musicoterapia, autoestima, violencia de género.

\section{Self-esteem, Music Therapy and Gender Violence A group meeting from the plurimodal approach}

ABSTRACT. In this article we present the results of the music therapy intervention, carried out with 24 women, victims of gender violence.

Objectives: Demonstrate that the use of music therapy contributes to a) To favor the increase of selfesteem of these women; b) improving interpersonal relationships; c) encourage the initiative

Method: For the sessions, we use the method of Plurimodal Approach of Music Therapy. To evaluate the results, were applied before and after, the Rosenberg Self-Esteem Scale and the Bruscia IAPS (the improvisation evaluation profiles) and to value initiative and interpersonal relationships.

Results: An increase of the self-esteem in all the women. It was observed that it was higher in those that were less time separated of their abuser. Likewise, through the IAPS it was observed that improving interpersonal relationships and that the initiative increased more, in the women who had been separated from their abuser more time.

Keywords: music therapy, self-esteem, gender violence.

Sumario. 1. Introducción.2. Método.3. Resultados 4. Discusión 5. Conclusiones 6. Referencias

Cómo citar. Guerrero, E. .(2019) Autoestima, Musicoterapia y Violencia de Género Un encuentro grupal desde el abordaje plurimodal.. Revista de Investigación en Musicoterapia, 2, 1-20 doi.org/10.15366/rim2019.3.001

\footnotetext{
${ }^{1}$ Universidad Católica “San Vicente Mártir empar.guerrero@ucv.es
} 


\section{Introducción}

La violencia de género se manifiesta por la desigualdad y "las relaciones de poder de los hombres sobre las mujeres, se ejerce sobre éstas por parte de quienes sean o hayan sido sus cónyuges o de quienes estén o hayan estado ligados a ellas por relaciones similares de afectividad, aun sin convivencia" (La Ley Orgánica 1/2004, p. 42168). En 1994, la Organización de las Naciones Unidas definió la violencia contra las mujeres como "todo acto de violencia basado en el género que tiene como resultado posible o real un daño físico, sexual o psicológico, incluidas las amenazas, la coerción o la privación arbitraria de la libertad ya sea que ocurra en la vida privada o en la pública”.

Existen varios tipos de maltrato: físico que se refleja en agresiones físicas infligidas de forma intencional con golpes, quemaduras, entre otros, causando lesiones corporales; psicológico que se lleva a cabo a través de humillaciones, insultos, amenazas y críticas exageradas y en muchas ocasiones realizadas públicamente desvalorizando a la mujer. Además, se incluyen otros comportamientos como un control excesivo sobre lo que hace esta, control del dinero, anulación en la toma de decisiones o aislamiento social; y sexual que se lleva a cabo con actos que atentan contra la libertad sexual de la persona y contra su dignidad a través de relaciones sexuales forzadas.

De estos tres, el abuso psicológico, es el que se considera más perjudicial para la víctima ya que es el más difícil de percibir por parte de la persona que lo recibe. Así mismo, las estadísticas consideran que este tipo de violencia es el más frecuente y es la que afecta de forma directa y consistente a la baja autoestima de las mujeres maltratadas (Alberdi y Matas, 2002).

Las mujeres que sufren maltrato de forma continuada, además de distorsiones cognitivas, pueden cambiar la forma de verse a sí mismas o de relacionarse con los demás, desarrollando síntomas del Trastorno de Estrés Postraumático (Romero, 2015), mostrando alta ansiedad, depresión, baja autoestima, miedo, dificultad en la regulación y expresión de sentimientos, dificultad en las estrategias de afrontamiento, sentimientos de impotencia y desesperanza, así como baja asertividad, (Echeburúa y Corral, 1998; Amor, et al. 2001; Martin, Taft y Resick 2007).

El énfasis en el abordaje y erradicación de la violencia de género se ha orientado principalmente a estudiar las secuelas de los episodios de violencia en las víctimas 
(Delgado, Sánchez, Fernández, 2012) y a intervenir con ellas desde diferentes ámbitos como el legal, laboral, social, psicológico, y musicoterapéutico. En este sentido (Cassity y Theobold, 1990; Whipple y Lindsey 1999; Curtis, 2016; Fernández, 2010; Parra, 2008; Lago, 2012; Wolfe, 2016; y Guerrero y Cruces, 2019), han demostrado que el uso de la musicoterapia incide positivamente en la mejora del área emocional de las mujeres víctimas de violencia de género.

En la mayoría de estos estudios el método musicoterapéutico utilizado, ha sido el Abordaje Plurimodal. Este método considera el ser humano como unidad biopsicosocioespiritual, reconociendo el ser humano de forma integral. Por tanto, el diagnóstico debe llevarse a cabo atendiendo todas las dimensiones del individuo (Schapira, 2007).

Este método ofrece gran diversidad de técnicas como, la improvisación musical terapéutica, el trabajo con canciones, la técnica de estimulación de imágenes y sensaciones a través del sonido, y la utilización de música editada, siendo adecuada para llevar a cabo intervenciones individuales y grupales.

La improvisación musical terapéutica es considerada un método primario de trabajo, a partir de la creencia de que el sonido que se hace nos representa. La improvisación musical será terapéutica en la medida que sea analizada a través de diferentes instrumentos.

Asimismo, las canciones son consideradas como materiales privilegiados puesto que forman parte de la historia de las personas desde que nacen. La creación, canto conjunto, improvisación, o inducción evocativa consciente, son algunas de las actividades que pueden introducirse cuando se trabaja con canciones.

En la estimulación de imágenes y sensaciones a través del sonido, se propone la audición de una secuencia de estímulos musicales, diseñada de manera artesanal por el musicoterapeuta para la situación singular del paciente o grupo.

Por último, en el uso selectivo de la música editada, se pueden utilizar temas musicales para caldear o facilitar una relajación, en la que la música "alcanza el estatus de objeto de transferencia y actúa como co-terapeuta subordinada a la implementación del profesional" (Schapira, 2007, p. 168).

La musicoterapia "no es sólo un arte, que como tal tienen que ver con la subjetividad, individualidad, creatividad y belleza, sino también una ciencia que supone objetividad, 
colectividad, repetición y verdad" (Poch, 2001, p. 42). En definitiva, la musicoterapia es el uso dosificado de la música en el tratamiento, la rehabilitación, la educación, reeducación y el adiestramiento de adultos y niños que padezcan trastornos físicos, mentales y emocionales.

Con el fin de continuar aportando nuevos datos sobre los beneficios de la musicoterapia con mujeres víctimas de violencia de género, se llevó a cabo la propuesta musicoterapéutica que se presenta. Los objetivos que se diseñaron fueron los siguientes: a) Favorecer el aumento de la autoestima de estas mujeres; b) Contribuir a mejorar las relaciones interpersonales; c) Fomentar la iniciativa y la toma de decisiones.

\section{Método}

\section{Participantes}

Las mujeres que participaron en el proceso musicoterapéutico tenían entre 22 y 56 años y todas habían sido víctimas de violencia de género. Fueron derivadas de tres asociaciones de la provincia de Valencia. Para llevar a cabo el estudio que se presenta, fueron distribuidas en tres grupos, de ocho mujeres cada uno. En cada grupo había cuatro mujeres que llevaban más de tres años, separadas de su maltratador y cuatro que se habían separado hacía menos de tres años. En lo que se refiere al nivel de estudios, en todos los grupos, había una mujer con estudios superiores y las demás tenían el título de Graduado Escolar, estudios de Formación Profesional o estudios de Bachiller.

\section{Procedimiento}

Con cada grupo, la intervención musicoterapéutica se llevó a cabo a lo largo de 12 sesiones, una a la semana, con una duración de 90 minutos. Dicha intervención tuvo lugar desde febrero hasta mayo de 2018, se desarrolló en grupo y se utilizaron técnicas del Método de Abordaje Plurimodal de Musicoterapia (Schapira, 2007).

El lugar de realización fue en la Sede San Juan Bautista de la Universidad Católica "San Vicente Mártir" en Valencia. La sala contaba con todo el material adecuado para el desarrollo de la intervención: instrumentos variados de pequeña percusión, como claves, panderetas, crótalos, cajas chinas, otros como una guitarra, dos palos de agua, un oceandrum, dos yembés, una cabassa, y dos triángulos. Además, la sala disponía de otros materiales como colchonetas, ordenador con conexión wi-fi, altavoces y sillas. 


\section{Recopilación de datos}

Para la recopilación de datos se contó con dos instrumentos diferentes: Escala de Autoestima de Rosenberg, y los Perfiles de Valoración de las Improvisaciones (IAPS) de Bruscia.

La Escala de Autoestima de Rosenberg, que se pasó antes y después de la intervención, permite explorar la autoestima personal entendida como los sentimientos de valía personal y de respeto a sí mismo. Consta de 10 ítems, de los que cinco están enunciados de forma positiva y cinco de forma negativa. Todos ellos se pueden valorar en escala Likert de A a D (A, muy de acuerdo y D, muy en desacuerdo). Los baremos son: Autoestima elevada (de 30 a 40 puntos); autoestima media (de 26 a 29 puntos); autoestima baja (menos de 25 puntos).

Perfiles de Valoración de las Improvisaciones (IAPS) de Bruscia. Este instrumento contempla 6 perfiles (integración, variabilidad, tensión, congruencia, saliencia y autonomía). Cada uno de estos perfiles contiene variables y gradientes, separadas para los diferentes elementos musicales y sus componentes. En nuestro estudio nos centramos en el perfil de "Autonomía", ya que con este perfil se pueden analizar relaciones intermusicales, relaciones interpersonales, así como la iniciativa y la toma de decisiones (Bruscia, 2007). Se llevó a cabo una valoración de la improvisación en la primera sesión y en la última, en cada uno de los grupos, con el fin de determinar las diferencias en toma de decisiones, la iniciativa y las relaciones intergruples. La valoración se hizo atendiendo a la variable rítmica (fuente sonora); variable tonal (armonía, melodía); volumen (intensidad); y variable programática (relaciones interpersonales).

\section{Sesiones de musicoterapia}

Las 12 sesiones se estructuraron en tres partes, en las que se incluía la acogida (bienvenida a través de una canción, un saludo, la expresión de un deseo para la sesión), la parte central en la que, se desarrollaba una de las técnicas del Abordaje Plurimodal (improvisación, trabajo con canciones, estimulación a través de imágenes o uso de la música editada) y cierre (valoración de la sesión). La propuesta musicoterapéutica era la misma para los tres grupos. 


\section{Análisis de los datos}

Para analizar los datos obtenidos a través de la Escala de Autoestima de Rosenberg se utilizaron tablas de Excel 2013. Para analizar la variable Autonomía del IAPS de Bruscia se registraron los comportamientos observados.

\section{Resultados}

Los resultados obtenidos en el pre-test y en el post-test, en la Escala de Autoestima de Rosenberg, en cada uno de los grupos de mujeres víctimas de violencia de género, se exponen en las gráficas siguientes (1-3).

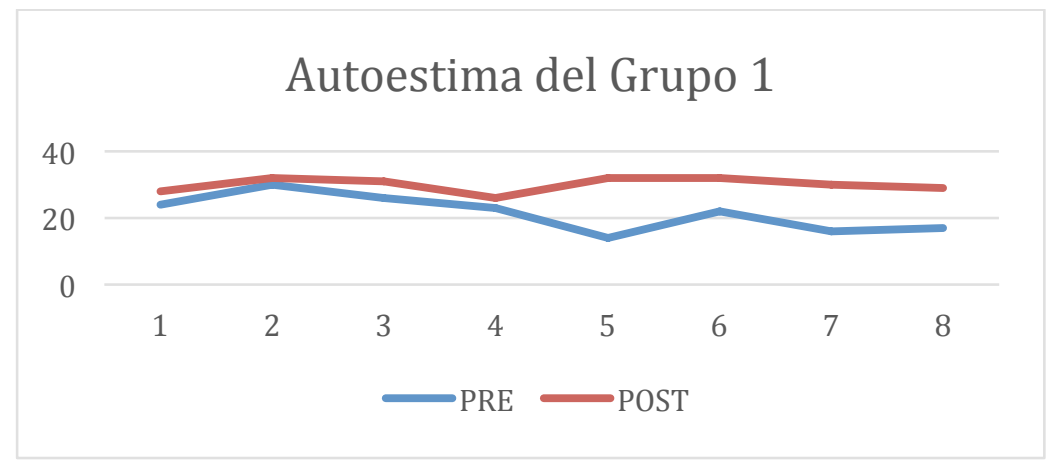

Figura 1. Pre-test y post-test del Grupo 1 (Escala de Autoestima de Rosenberg)

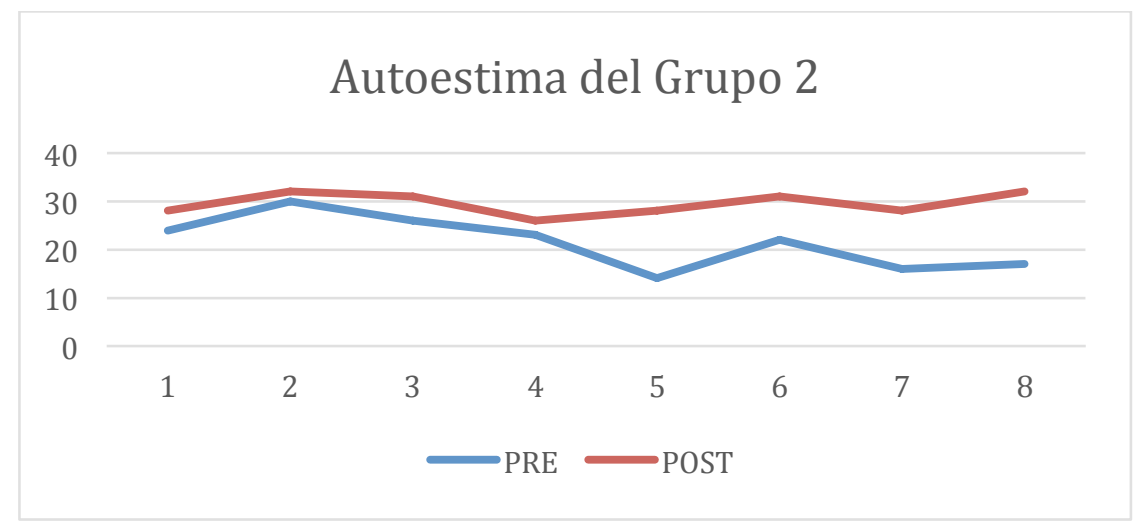

Figura 2. Pre-test y post-test del Grupo 2 (Escala de Autoestima de Rosenberg) 


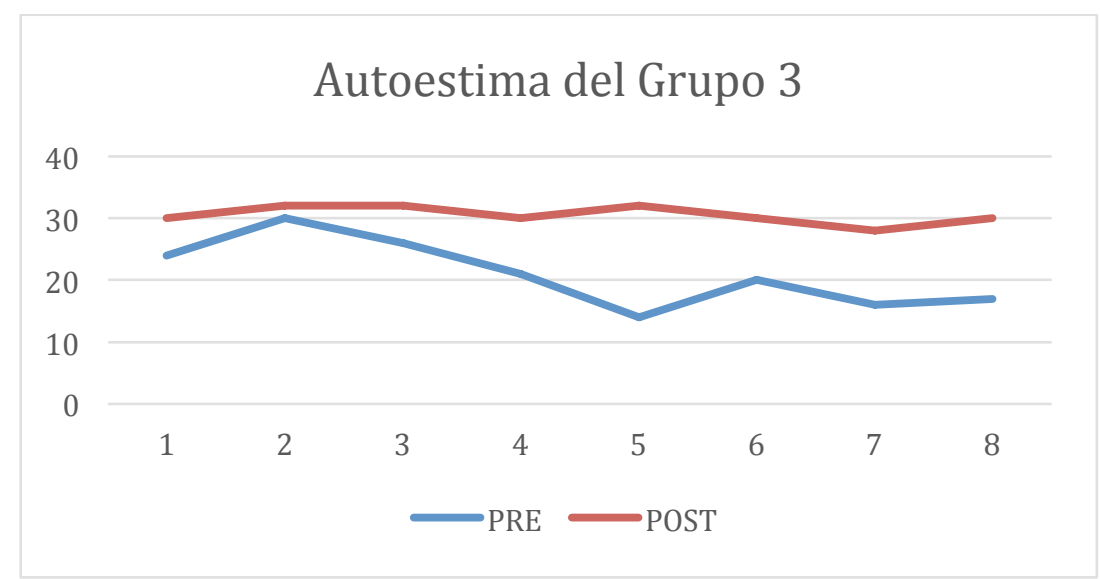

Figura 3. Pre-test y post-test del Grupo 3 (Escala de Autoestima de Rosenberg)

En los tres grupos, de las ocho mujeres representadas en cada gráfica, las cuatro primeras llevaban, más de tres años separadas de su maltratador, mientras que las cuatro últimas eran mujeres que llevaban menos de tres años.

A continuación, (FIGURA 4), se exponen los resultados obtenidos en la Escala de Rosenberg, en el pre-test y en el post-test, de las 12 mujeres que llevaban, menos de tres años separadas del maltratador.
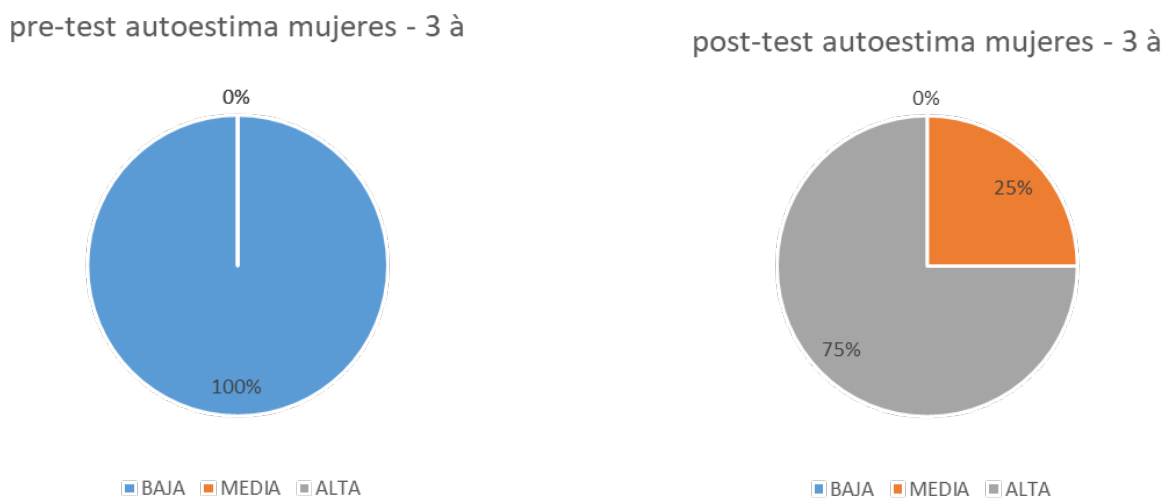

Figura 4. $\mathrm{MVVG}^{\mathrm{o}}$ que llevan menos de 3 años separadas del maltratador

En la Figura 5, se muestran los resultados del pre-test y post-test sobre la autoestima de las 12 mujeres que ya estaban separadas de su maltratador hacía más de tres años. 


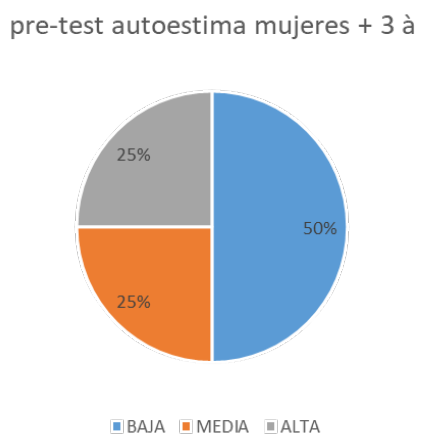

post-test autoestima mujeres +3 à

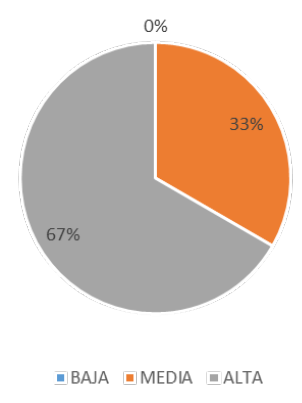

Figura 5. Figura 5.MVVG ${ }^{\circ}$ que llevan más de 3 años separadas del maltratador

Otro aspecto que se valoró en la intervención fue el tipo de respuesta que las mujeres mostraron en las improvisaciones, utilizando el IAPS de Bruscia. En las siguientes tablas (1-4) se muestra el resultado grupal. Para ello, de los 6 perfiles, se tuvo en cuenta el de autonomía, con las variables rítmica; tonal (armonía, melodía); volumen (intensidad); y Programática (expresiones y relaciones intergrupales).

Tabla 1

Resultado de la variable rítmica en la $1^{a}$ y $12^{a}$ sesión

Variable Rítmica

\begin{tabular}{|c|c|c|}
\hline & Sesión 1 & Sesión 12 \\
\hline Grupo 1 & 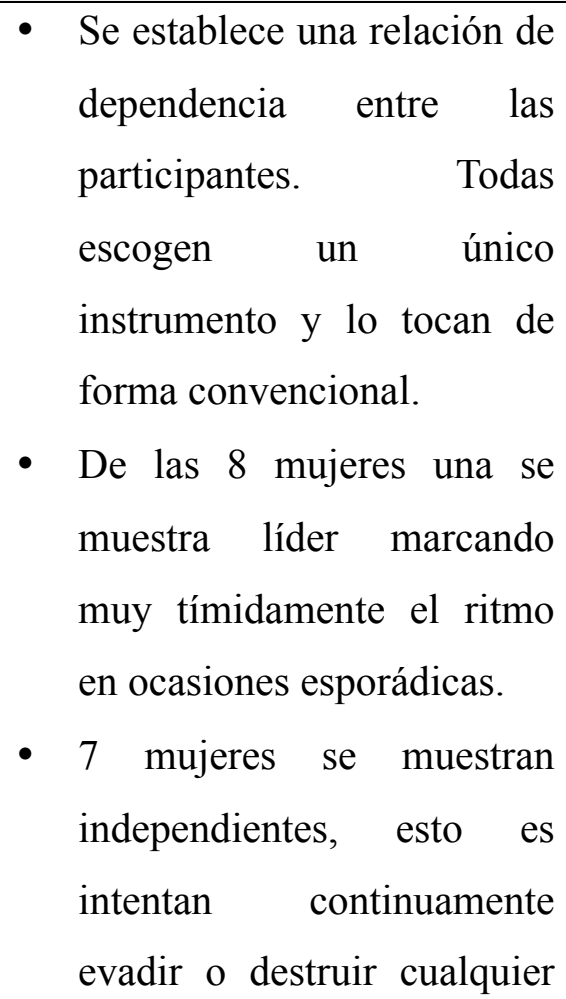 & $\begin{array}{l}\text { - Se crea una relación de } \\
\text { complementariedad en la } \\
\text { que sin mediar palabra van } \\
\text { cambiando de rol. Van } \\
\text { pasando de líder a } \\
\text { compañera, acompañando a } \\
\text { las demás. } \\
\text { - mujeres repiten más veces } \\
\text { como líder coincidiendo con } \\
\text { llevar más de tres años } \\
\text { separadas. } \\
2 \text { mujeres tienden a la } \\
\text { dependencia (coinciden con } \\
\text { llevar menos de } 3 \text { años }\end{array}$ \\
\hline
\end{tabular}




\begin{tabular}{|c|c|c|}
\hline & $\begin{array}{l}\text { relación grupal. } \\
\text { - A penas se observa } \\
\text { variación rítmica. }\end{array}$ & $\begin{array}{l}\text { separadas). No asumen en } \\
\text { ningún momento el rol de } \\
\text { líder, aunque sí de } \\
\text { acompañantes. } \\
\text { - Hay variación rítmica cada } \\
\text { vez que van cambiando de } \\
\text { líder. Se cambia de ritmo en } \\
\text { diferentes ocasiones. }\end{array}$ \\
\hline Grupo 2 & 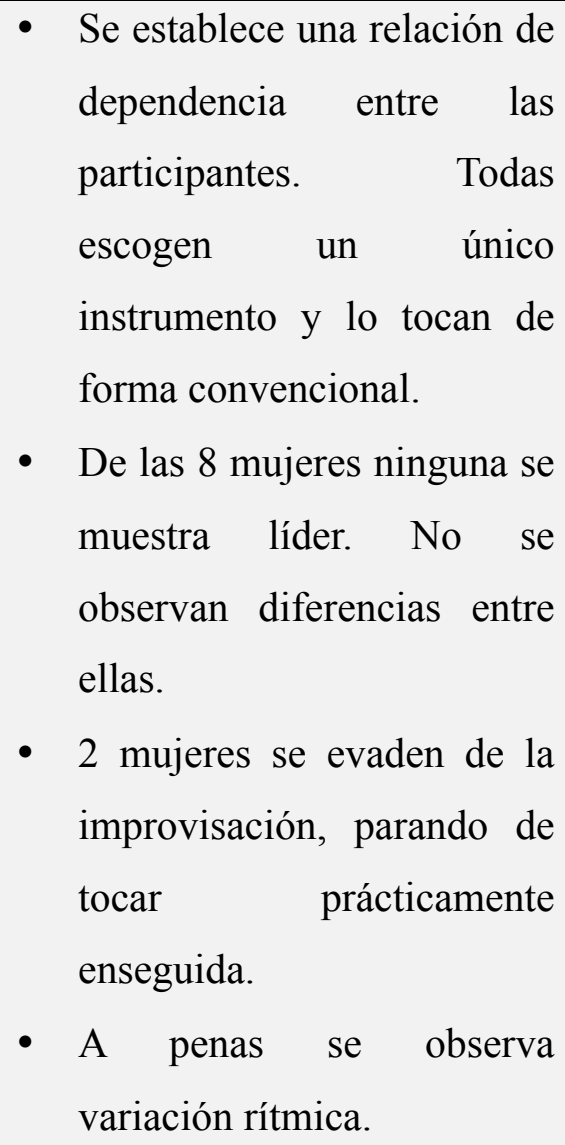 & $\begin{array}{l}\text { - Se crea una relación de } \\
\text { complementariedad. En este } \\
\text { grupo } 4 \text { mujeres ejercen con } \\
\text { más fuerza y en más } \\
\text { momentos de } \\
\text { improvisación, el rol de } \\
\text { líder. Coinciden con llevar } \\
\text { más de tres años separadas. } \\
\text { - de las mujeres se muestra } \\
\text { independiente de modo que } \\
\text { no sigue el ritmo de otras } \\
\text { compañeras. } \\
\text { Hay variación rítmica cada } \\
\text { vez que van cambiando de } \\
\text { líder. Se cambia de ritmo en } \\
\text { diferentes ocasiones }\end{array}$ \\
\hline Grupo 3 & 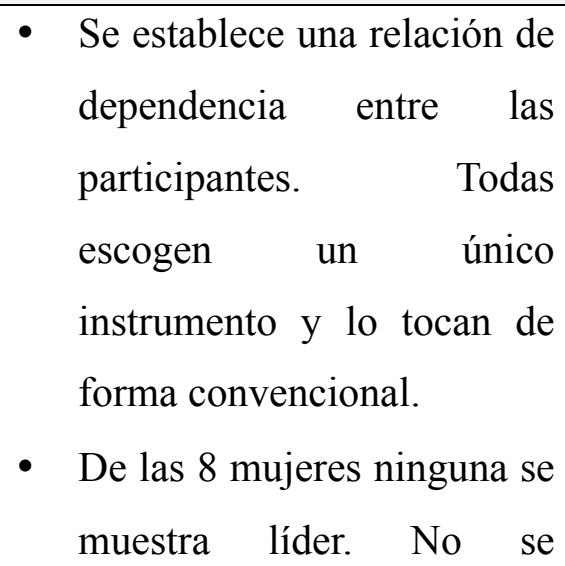 & $\begin{array}{l}\text { - Se crea una relación de } \\
\text { complementariedad. El } \\
\text { liderazgo va cambiando } \\
\text { entre } 4 \text { mujeres, de las } \\
\text { cuales } 3 \text { llevan más de tres } \\
\text { años separadas y una, } \\
\text { menos de tres años. } \\
\text { - Todas se muestran }\end{array}$ \\
\hline
\end{tabular}




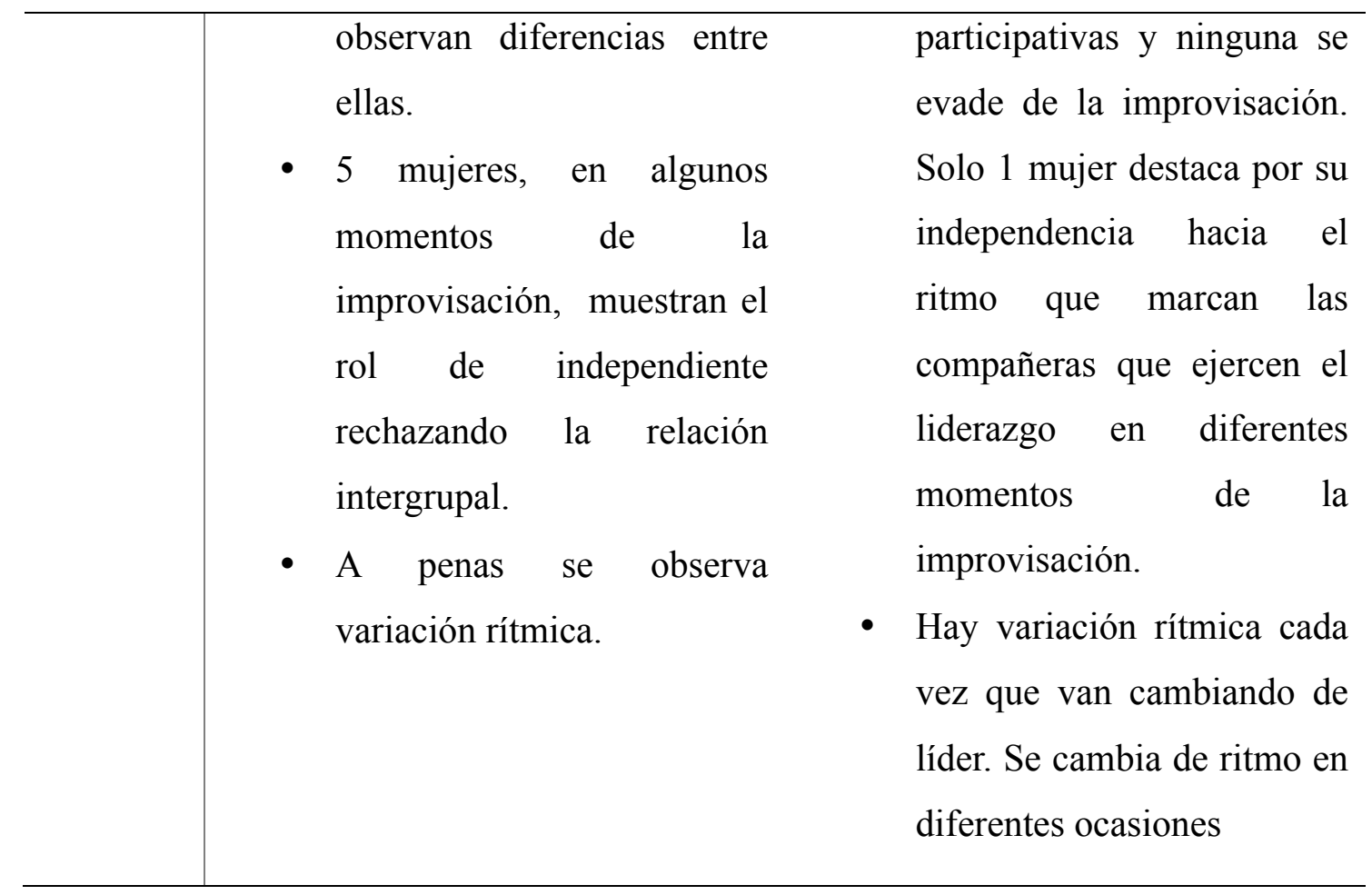

Tabla 2

Resultado de la variable tonal en la $1^{a}$ y $12^{a}$ sesión

Variable tonal (armonía, melodía)

\begin{tabular}{l|ccc}
\hline Grupo & Sesión 1 & \multicolumn{2}{c}{ Sesión 12 } \\
\hline Grupo 1 & - No surge ninguna melodía. & Hay variedad melódica, \\
& No hay secuencia de temas & sobre todo cuando 3 \\
melódicos. 1 mujer presenta & mujeres que ejercen el rol \\
una propuesta melódica & de líder presentan nuevas \\
pero no es seguida por las & propuestas. Se observa que \\
demás. & 2 mujeres completan \\
& algunas propuestas \\
& melódicas, enriqueciendo la \\
& improvisación. Hay 2 \\
& mujeres que introducen un \\
& instrumento más, cada una, \\
& para ofrecer nuevas \\
& melodías.
\end{tabular}




\begin{tabular}{|c|c|c|}
\hline Grupo 2 & $\begin{array}{l}\text { - No surge ninguna melodía. } \\
\text { No hay secuencia de temas } \\
\text { melódicos. } 2 \text { mujeres no } \\
\text { establecen ninguna relación } \\
\text { con las compañeras. }\end{array}$ & $\begin{array}{l}\text { - Hay gran variedad } \\
\text { melódica, creándose un } \\
\text { intercambio melódico entre } \\
\text { las participantes. Todas } \\
\text { introducen algún cambio } \\
\text { melódico a lo largo de las } \\
\text { sesiones, pero las } 4 \text { mujeres } \\
\text { que llevan más de } 3 \text { años } \\
\text { separadas toman más veces } \\
\text { la iniciativa de cambiar. }\end{array}$ \\
\hline Grupo 3 & $\begin{array}{l}\text { - No surge ninguna melodía. } \\
\text { No hay secuencia de temas } \\
\text { melódicos. }\end{array}$ & $\begin{array}{l}\text { - Hay gran variedad } \\
\text { melódica, creándose un } \\
\text { intercambio melódico entre } \\
\text { las participantes. Todas } \\
\text { introducen algún cambio } \\
\text { melódico a lo largo de las } \\
\text { sesiones, pero } 3 \text { mujeres } \\
\text { que llevan más de } 3 \text { años } \\
\text { separadas, toman más veces } \\
\text { la iniciativa de cambiar. }\end{array}$ \\
\hline
\end{tabular}

Tabla 3

Resultado de la variable volumen (intensidad) en la $1^{a}$ y $12^{a}$ sesión

Variable volumen (intensidad)

\begin{tabular}{l|ccl}
\hline Grupo & Sesión 1 & \multicolumn{2}{c}{ Sesión 12 } \\
\hline Grupo 1 & La improvisación es poco & A lo largo de la \\
& intensa. Los instrumentos & improvisación el volumen y \\
& son, en su mayoría de & la intensidad aumenta. No \\
& pequeña percusión. Ninguna & siempre la que ejerce de \\
& mujer varía la intensidad. Se & líder es la que toca con \\
& observa que 3 tocan con & mayor intensidad. Se \\
& menos intensidad que el & observa que hay 3 mujeres \\
\hline
\end{tabular}




\begin{tabular}{|c|c|c|}
\hline & resto. & $\begin{array}{l}\text { que propician el cambio en } \\
\text { el volumen y controlan } \\
\text { dichos cambios ejerciendo } \\
\text { de líder y de acompañantes. } \\
\text { Coinciden con ser mujeres } \\
\text { que llevan más de } 3 \text { años } \\
\text { separadas. }\end{array}$ \\
\hline Grupo 2 & $\begin{array}{l}\text { - La improvisación es poco } \\
\text { intensa y muy suave. No } \\
\text { hay prácticamente cambios } \\
\text { y el volumen es muy bajo. } \\
\text { Cuando dos mujeres dejan } \\
\text { de tocar el volumen } \\
\text { disminuye hasta hacerse el } \\
\text { silencio. }\end{array}$ & $\begin{array}{l}\text { - Hay muchas variaciones en } \\
\text { la intensidad. Hay } 4 \text { mujeres } \\
\text { que asumen el liderazgo en } \\
\text { repetidas ocasiones } \\
\text { controlando el volumen de } \\
\text { las improvisaciones. No } \\
\text { obstante se observa que } \\
\text { otras } 2 \text { mujeres proponen } \\
\text { cambios en la intensidad y } \\
\text { este es respetado, } \\
\text { intentando llegar a un } \\
\text { encuentro entre todas. }\end{array}$ \\
\hline Grupo 3 & $\begin{array}{l}\text { - La improvisación es poco } \\
\text { intensa. Los instrumentos } \\
\text { son, en su mayoría de } \\
\text { pequeña percusión. }\end{array}$ & 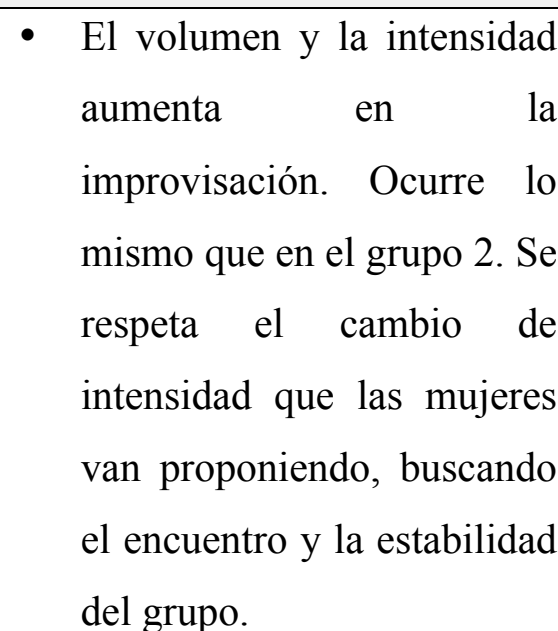 \\
\hline
\end{tabular}


Tabla 4

Resultado de la variable programática en la $1^{a}$ y $12^{a}$ sesión

Variable Programática (expresiones y relaciones intergrupales)

\begin{tabular}{|c|c|c|}
\hline Grupo & Sesión 1 & Sesión 12 \\
\hline Grupo 1 & $\begin{array}{l}\text { - Ninguna toma la iniciativa a } \\
\text { la hora de mostrar su idea. } \\
\text { Se esperan a que las } \\
\text { compañeras hablen y se } \\
\text { apoyan en lo que han dicho } \\
\text { las demás. Al final de la } \\
\text { improvisación todas han } \\
\text { aportado alguna idea. }\end{array}$ & $\begin{array}{l}\text { - Las ideas surgen } \\
\text { espontáneamente, pero } \\
\text { siempre toman la iniciativa } \\
3 \text { de las } 8 \text { mujeres. Son las } \\
\text { que llevan más de } 3 \text { años } \\
\text { separadas del maltratador. } \\
\text { - Se crea una relación grupal } \\
\text { de intercambio de ideas y } \\
\text { propuestas. }\end{array}$ \\
\hline Grupo 2 & $\begin{array}{l}\text { - Ninguna toma la iniciativa a } \\
\text { la hora de mostrar su idea. } 2 \\
\text { mujeres no muestran } \\
\text { ninguna idea. }\end{array}$ & $\begin{array}{l}\text { - Las ideas surgen } \\
\text { espontáneamente, pero hay } \\
1 \text { mujer que prácticamente } \\
\text { no interactúa con las } \\
\text { propuestas de } \\
\text { compañeras. }\end{array}$ \\
\hline Grupo 3 & $\begin{array}{l}\text { - } 1 \text { mujer toma la iniciativa } \\
\text { de forma espontánea. Esto } \\
\text { sirve para que otras } 3 \\
\text { continúen apoyándose en lo } \\
\text { dicho. }\end{array}$ & $\begin{array}{l}\text { - } 4 \text { mujeres destacan en } \\
\text { proyectar nuevas ideas. } 3 \\
\text { llevan más de } 3 \text { años } \\
\text { separadas y } 1 \text { menos de } 3 . \\
\text { El resto de participantes } \\
\text { interactúan aceptando las } \\
\text { propuestas de las } \\
\text { compañeras. }\end{array}$ \\
\hline
\end{tabular}

\section{Análisis de los resultados}

De los resultados obtenidos en la Escala de Autoestima de Rosenberg, se puede destacar el aumento considerable de la autoestima de todas las mujeres. En cuanto al análisis por 
grupos, se observan diferencias significativas en las mujeres que llevaban menos de años separadas de su maltratador.

En el grupo 1, (FIGURA 1), en el pre-test, 6 mujeres presentan una autoestima baja (1624 puntos), 1 mujer, autoestima media (26 puntos), y 1, autoestima alta, (30 puntos). En el post-test se observa que todas las mujeres, han aumentado la puntuación, siendo el resultado: 3 mujeres autoestima media, (26-29 puntos), y 5 autoestima alta (30-32 puntos). Las diferencias más significativas se producen en las mujeres que llevaban, menos de 3 años, separadas de su maltratador. Las 4 inician el proceso terapéutico con una autoestima muy baja (14-22 puntos). Al finalizar el proceso 1 de estas mujeres puntúa (29), autoestima media y 3 obtienen (30-32 puntos), autoestima alta. En las otras 4 mujeres, aunque sí hay un aumento en la autoestima, las diferencias no son estadísticamente significativas.

En el grupo 2, (FIGURA 2) en el pre-test se obtienen los mismos resultados que en el grupo anterior. 6 mujeres presentan una autoestima baja, 1 autoestima media y otra autoestima alta. En el post-test las puntuaciones que se obtienen son: 4 mujeres presentan autoestima media (26-28 puntos) y 4 presentan autoestima alta (31-32 puntos). Igual que en el anterior grupo se observa una diferencia significativa en las 4 mujeres que llevaban, menos de 3 años, separadas de su maltratador.

En el grupo 3, (FIGURA 3), al iniciar el proceso musicoterapéutico 6 mujeres puntúan (14-24 puntos) siendo una autoestima baja. 1 presenta autoestima media (28) y 1 autoestima alta (30). Cuando finaliza el proceso, en el post-test, 9 mujeres presentan una autoestima alta (30-32 puntos) y 1 mujer presenta autoestima media (28). En este grupo las diferencias entre el pre y post-test son mayores que en los otros dos grupos. Se conserva la tendencia de los dos grupos anteriores, en los que se produce un cambio más significativo en las 4 mujeres que llevaban menos de 3 años, separadas de su pareja.

Si analizamos los resultados del pre-test y post-test de las mujeres que llevaban menos de 3 años, separadas de su maltratador (FIGURA 4) se observa una diferencia significativa entre los resultados al inicio del proceso y al final. De las 12 mujeres, el $100 \%$ empiezan con una autoestima baja. Al finalizar el proceso, el 75\% presenta una autoestima alta y el $25 \%$ media. 
En las 12 mujeres que llevaban más de 3 años separadas de su maltratador, (FIGURA 5), se muestran cambios en la autoestima pero, estadísticamente, menos significativas que en el grupo anterior. Al inicio, el $50 \%$ de las mujeres, presentaban una autoestima baja, el $25 \%$ autoestima media y el $25 \%$ autoestima alta. Al finalizar el proceso musicoterapéutico, el 67\% muestran una autoestima alta, y el 33\% una autoestima media. Ninguna muestra una autoestima baja.

Los resultados obtenidos en el análisis de las improvisaciones, centrado en el perfil de autonomía, y atendiendo a las variables del IAPS de Bruscia, demuestran que existe un aumento en cuanto a la toma de decisiones siendo, estadísticamente, más significativo, en mujeres que llevaban más de tres años separadas de su maltratador. Esta variable también permite observar las relaciones intermusicales e intergrupales.

En lo que respecta a la variable rítmica, (TABLA 1), en la primera improvisación, en los tres grupos, se establece una relación de dependencia entre las mujeres, esperando la aceptación de la musicoterapeuta y que sea ésta la que cambie o marque el ritmo. El $50 \%$ de mujeres asumen, en algunos momentos de la improvisación, el rol de independiente, esto es, rechazan cualquier relación grupal con las compañeras o se evaden sin tener en cuenta el ritmo que se va generando. Sólo 1, de las 24 mujeres, muestra el perfil de líder, en un momento puntual de la improvisación. En la improvisación no se muestra variación rítmica.

En la última sesión se observa que se crea una relación de complementariedad en los 3 grupos. De las 24 mujeres, 11 asumen el rol de líder en diferentes momentos de la improvisación, sirviendo de sostén rítmico, o cambiándolo en diferentes ocasiones. Todas ellas llevaban más de 3 años separadas del maltratador. A su vez, se observa que, en los 3 grupos, sólo 2 mujeres se muestran independientes (se evaden y no se interrelaciona) y también disminuye el nivel de dependencia, cambiando al rol de compañeras (de complementariedad). En esta última improvisación, se muestra una mayor variación rítmica provocada por las propias mujeres,

En cuanto a la variable tonal, (TABLA 2), en la primera sesión, no surge ninguna secuencia melódica, ni cambio tonal. No existe complementariedad y no surge ninguna armonía. En la última sesión, se produce una gran variedad melódica, variando el tono y las propuestas melódicas. Se crea una relación de dependencia siguiéndose unas a otras y destacan 10 mujeres que asumen el rol de líder, provocando cambios en el tono y 
melodía y de ellas, 3 deciden introducir otros instrumentos diferentes a los escogidos al principio. Estas 10 mujeres llevaban más de 3 años separadas de su maltratador.

En la siguiente variable, que es el volumen (TABLA 3), en la primera sesión, en los 3 grupos, se produce una improvisación poco intensa y suave con ningún cambio. En general las mujeres se muestran inseguras con respecto a lo que deben hacer, tocan de forma suave el instrumento y con muy poca intensidad. En la sesión $n^{\circ} 12$ el volumen aumenta, variando a lo largo de la improvisación. Se producen momentos de una gran intensidad, generado por las mujeres que ejercen liderazgo y que son seguidas por otras mujeres. Se observa que todas buscan una interrelación, se buscan con la mirada y cuando aumenta la intensidad surge una gran complementariedad grupal y relación intermusical.

La variable programática (TABLA 4), permite analizar las relaciones de rol que las mujeres establecen con las compañeras, en el desarrollo del programa y en las improvisaciones. En la primera sesión prácticamente ninguna toma la iniciativa a la hora de mostrar ideas o compartir experiencias de forma espontánea. En la última sesión las mujeres que llevan más de 3 años separadas de su maltratador, son las primeras en aportar sus ideas, cómo se sienten, y refuerzan lo que las demás compañeras añaden. Se observa que existe una interacción grupal y un intercambio de ideas entre ellas.

\section{Discusión}

Existen diferentes estudios que analizan la incidencia de la musicoterapia en la autoestima de las mujeres víctimas de violencia de género (Fernández, 2006; Parra, 2008; Lago, 2012). Todos ellos han confirmado resultados positivos y han demostrado que se obtienen mejores resultados cuando la intervención es grupal. Pero en ninguno de los estudios revisados se contemplaba la variable que se ha tenido en cuenta en este proyecto, el tiempo de separación de su maltratador.

La intervención se llevó a cabo de forma grupal, puesto que los estudios (Santandreu, et al, 2014), han demostrado, que en mujeres víctimas de violencia de género, se obtienen mejores resultados si se interviene grupalmente.

En la intervención musicoterapéutica, se han utilizado las técnicas propias del abordaje Plurimodal, ya que ofrecen gran variedad de posibilidades. La experiencia nos ha 
demostrado que es un método totalmente adecuado para trabajar con estas mujeres por los resultados que hemos obtenido en las diferentes intervenciones.

Cabe destacar, que también se ha podido demostrar que ha aumentado el nivel de autoestima en todas las mujeres, y que este aumento es mayor en las que llevaban menos de 3 años, separadas de la pareja. El maltrato tiene como consecuencia, una baja autoestima, dificultad en la regulación de sentimientos y emociones, y otras consecuencias relacionadas con el área emocional (Alberdi y Matas, 2002), que se ve afectada debido al maltrato continuado. Por ello, se hace necesaria una intervención que favorezca la recuperación de esta área y la musicoterapia demuestra que es válida para favorecer esa mejora.

Para alcanzar este objetivo se ha utilizado sobre todo el trabajo con canciones con todas las posibilidades que Schapira (2007) plantea, o la utilización de la estimulación de imágenes y sensación a través del sonido.

Otro aspecto que debe ser abordado cuando se interviene con mujeres víctimas de violencia de género, son las relaciones intergrupales, ya que una de las consecuencias que la violencia de género tiene sobre la mujer, es el aislamiento (Muñoz, 2015). En el proyecto se planteaba el objetivo de favorecer las relaciones intergrupales con el fin de crear una red de apoyo y confianza entre las mujeres. Este objetivo también se ha conseguido a lo largo de las sesiones, y ello se constata en la valoración que se hace atendiendo a los criterios del IAPS de Bruscia. En la última sesión se observa que existe una gran complementariedad entre las mujeres acompañándose en la improvisación realizada.

Asimismo se ha constatado que a través de la musicoterapia se favorece el aumento en la toma de decisiones e iniciativa. En este sentido, se observó que las mujeres que llevaban más de 3 años separadas de su pareja tomaban más la iniciativa y asumían el rol de líder en más ocasiones, cambiando el volumen y la intensidad de la improvisación o marcando ritmos diferentes. Estas mismas mujeres, a medida que el proceso terapéutico avanzaba, eran las primeras en expresar ideas o proponer alternativas.

Si se tienen en cuenta los resultados obtenidos en este estudio y las investigaciones anteriores, relacionadas con musicoterapia y mujeres víctimas de violencia de género, se puede afirmar que la utilización de la musicoterapia es una opción totalmente adecuada 
para favorecer el aumento de la autoestima, la toma de decisiones y fomentar las relaciones intergrupales en mujeres que hayan sufrido maltrato.

\section{Conclusiones}

La musicoterapia tiene efectos positivos en mujeres maltratadas, tal y como demuestran estudios anteriores a este. Con ella se puede incidir en diferentes como el aumento de la confianza, la autoestima o la capacidad para tomar decisiones, entre otros. Con este estudio se pretende continuar aportando experiencias científicas respecto al uso de la musicoterapia, y demostrar que, con ella, se puede mejorar el área emocional de las mujeres víctimas de violencia de género.

Se aboga por un proceso musicoterapéutico que complemente otro tipo de intervenciones, ya que el trabajo con estas mujeres debe abordarse de forma interdisciplinar, teniendo en cuenta que las consecuencias del maltrato son múltiples y que deben ser atendidas por diferentes profesionales.

No obstante, a pesar de los resultados obtenidos, cabe afirmar que son muchos los aspectos que quedan por estudiar en este ámbito, (resultados en la intervención individual, aplicación de otros modelos, mejora de otras competencias emocionales, etc.), y por ello es necesario continuar profundizando en la aplicación de la musicoterapia en mujeres víctimas de violencia de género.

\section{Referencias bibliográficas}

Alberdi I. y Matas N. (2002). La violencia doméstica. Informe sobre los malos tratos a mujeres en España. Barcelona: Fundación "La Caixa"

Amor P., Echeburúa E., Corral P., Sarasúa B., \& Zubizarreta I., (2001). Maltrato físico y maltrato psicológico en mujeres víctimas de violencia en el hogar: un estudio comparativo. Revista Internacional de Psicología Clínica y de la Salud, 2, 227246. https://doi.org/10.5944/rppc.vol.6.num.3.2001.3913

Bruscia, K.E. (2007). Musicoterapia. Métodos y prácticas. México D.F.: Pax México

Cassity, M. y Theobold, K. (1990). Domestic Violence: Assessments and treatments employed by music therapists. Journal of Music Therapy, 27(4), 179-194. https://doi.org/10.1093/jmt/27.4.179 
Curtis, S. (2016). Music Therapy for Women who have experienced domestic violence. The Oxford Handbook of Music Therapy, (289-298). Oxford University Press. http://www.oxfordhandbooks.com/view/10.1093/oxfordhb/9780199639755.001. 0001/oxfordhb-9780199639755-e-30?print=pdf

Delgado, M.C., Sánchez, M.C., Fernández-Dávila, P. (2012). Atributos y estereotipos de género asociados al ciclo de la violencia contra la mujer. Universitas Psychologica, 11(3), 778. https://doi.org/10.11144/Javeriana.upsy11-3.aega

Echeburúa, E. y Corral, P. (1998a). Trastorno de estrés postraumático. En M.A. Vallejo, Manual de terapia de conducta (Vol. 1, pp. 429-471). Madrid: Dykinson.

Fernández, T. (2006). Hacia un nuevo camino: programa de Investigación-acción sobre autoestima y musicoterapia con mujeres violentadas. En Enseñanza $e$ investigación en psicología, enero-junio, año/vol. 11, 001. Méjico: Universidad Veracruzana. pp. 65-79. https://www.redalyc.org/articulo.oa?id=29211105

Fernández, T. (2010). Musicoterapia y género: Una nueva visión en el trabajo de reconstrucción de la identidad en mujeres que padecen violencia doméstica y ante determinadas situaciones de salud. Revista electrónica Sistema Mexicano de Investigación en psicología. http:/Www.academia.edu/1967588/

Guerrero, E., \& Cruces, C. (2019). Mujeres víctimas de violencia de género y musicoterapia. Una experiencia grupal desde un abordaje plurimodal. RizomaTrans 2019. Catarroja: Edicions Florida

Lago, P. (2012). La musicoterapia en el trabajo de mejora personal de un grupo de mujeres maltratadas. IV Congreso Nacional de Musicoterapia. Madrid: fundación musicoterapia $\quad \mathrm{y}$ salud, pp.59-79. https://www.academia.edu/28704010/ACTAS_IV_CONGRESO_NACIONAL_ DE_MUSICOTERAPIA

Ley Orgánica 1/2004, de 28 de diciembre de Medidas de protección integral contra la violencia de género. $\mathrm{BOE} \mathrm{N}^{\mathrm{o}} 313,29$ de diciembre de 2004. https://www.boe.es/buscar/pdf/2004/BOE-A-2004-21760-consolidado.pdf

Martin, E.K., Taft, C.T. y Resick, P.A. (2007). A review of marital rape. Aggression and Violent $\quad$ Behavior, 329-347. https://www.researchgate.net/profile/Patricia_Resick2/publication/223831051_A _review_of_marital_rape/links/5bf2f4ad299bf1124fde58c0/A-review-ofmarital-rape.pdf 
Muñoz, C. (2015). La violencia de género. Identificación y prevención. Amazon: Germany

Parra, C. (2008). L'aplicació de la musicoterapia en el col-lectiu de dones maltractades. (Tesi doctoral). Facultat de Psicologia, Ciències de l'Educació. Barcelona: Universitat Ramon Llull. https://www.tdx.cat/handle/10803/9265

Poch Blasco, S. (2001). Importancia de la musicoterapia en el área emocional del ser humano. Revista Interuniversitaria de Formación del Profesorado(12), 91-113. https://www.redalyc.org/html/274/27404208/

Romero, I (Coord.) (2015). Guía de intervención en casos de violencia de género. Una mirada para ver. Madrid: Ed. Síntesis

Santandreu, M., Torrents, L., Rosalia, R., Iborra, A. (2014). Violencia de género y autoestima: efectividad de una intervención grupal. Apuntes de Psicología, vol. 32, 1, 57-63. file:///C:/Users/Usuario/AppData/Local/Temp/487-1124-1-PB1.pdf

Schapira, D.; Ferrari, K.; Sánchez, V.; Hugo, M. (2007). Musicoterapia. Abordaje plurimodal. Argentina: ADIM Ediciones.

Wolfe J. C. (2016). The Impact of Music Therapy on Self-Perceived Levels of Independence for Female Survivors of Abuse (Doctoral dissertation, Ohio University).

https://etd.ohiolink.edu/!etd.send file?accession=ohiou1462406390\&disposition $=$ inline 